\title{
Development of a UAV-mounted light source for fluorescence detector calibration of the Telescope Array experiment
}

\author{
Motoki HaYashi $^{1}$ and Yuichiro TAmeda ${ }^{2}$ Takayuki Tomida $^{1}$ Yoshiki Tsunesada $^{3}$ \\ Terutsugu SEKI ${ }^{1}$ Yoshinori SAITO ${ }^{1}$ and for the Telescope Array Collaboration \\ ${ }^{1}$ Shinshu University, Nagano, Nagano, Japan \\ ${ }^{2}$ Kanagawa University, Yokohama, Kanagawa, Japan \\ ${ }^{3}$ Osaka City University, Osaka, Osaka, Japan \\ E-mail:15tm524j@shinshu-u.ac.jp
}

(Received April 17, 2017)

We are developing a unmanned aerial vehicle (UAV), which is called "Opt-copter", carrying a calibrated light source for fluorescence detector (FD) calibration of the Telescope Array (TA) experiment. Opt-copter is equipped with a high accuracy GPS device and a LED light source in the shape of a dodecahedron. A positioning accuracy of the GPS mounted on the $\mathrm{UAV}$ is $0.1 \mathrm{~m}$, which meets the requirement for the calibration of the FDs at the distance of $100 \mathrm{~m}$. The light source consists of 12 UV LEDs attached on each side of the dodecahedron, and it is covered with a spherical diffuser to improve the spatial uniformity of the light intensity. We report the status of Opt-copter development and the results of its test at the TA site.

KEYWORDS: Telescope Array, UAV, Calibration

\section{Introduction}

The TA observatory has performed the observation of ultra high energy cosmic rays (UHECRs) with energy above $10^{18} \mathrm{eV}$ using hybrid technique with fluorescence detectors (FDs) and surface detectors (SDs) array in Utah, U.S [1]. Motivation of Opt-copter is to calibrate all of the FDs by a single device, which can improve the systematic uncertainty such as a energy scale difference between the FDs at the different site (Black Rock, Long Ridge site). Accordingly, we are planning to calibrate by a common standard light source which is easy to move like a radio-controlled helicopter (Auger has also already developed a similar device [2] [3].) At the TA site, we have already been testing the light source.

\section{Opt-copter}

In order to put the light source within the field of view of the FDs to calibrate all of the pixels of FDs, the calibration device requires flight performance. Portability such that the device can be mounted on passenger cars is also required to calibrate each FDs, because FDs are separated by a distance of $35 \mathrm{~km}$. For sensitivity calibration, the calibration light source should be uniform and stable. High precision GPS is used to guarantee positioning accuracy in the atmosphere. Therefore, we developed a calibration device equipped with a uniform and stable light source and high precision GPS on Unmanned Aerial Vehicle (UAV), and call it Opt-copter. The calibration concept and assembled Opt-copter are shown in Fig. 1 and 2. 


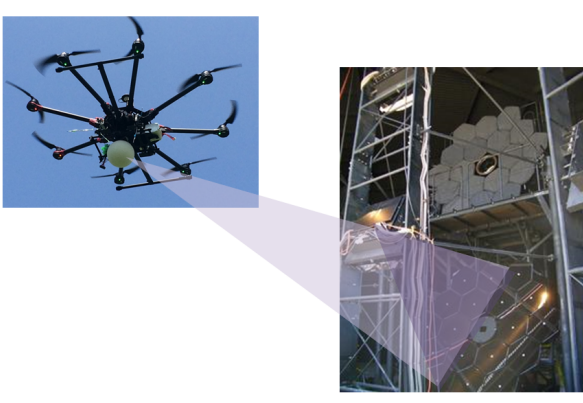

Fig. 1. Diagram of FD calibration using Optcopter.

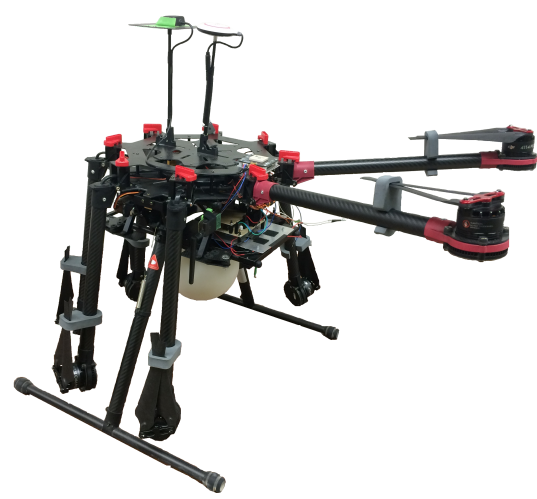

Fig. 2. The arms of Opt-copter can be folded which realize high portability. The light source is installed under the body.

The goal of Opt-copter is to improve the systematic uncertainty of FD PMT gain to $\pm 2 \%$. Therefore, it is required that the stabilities of light intensity and position determination are $\pm 2 \%$ and $\pm 10 \mathrm{~cm}$ respectively.

\section{Configuration of Opt-copter}

Opt-copter consists of the following items.

[UAV] The base of the UAV is a multicopter with 8 rotors (DJI, S1000+). With integrated design, UAV is simple in construction, easy to assemble, flight is stable and easy to control. Using a lithium-ion polymer secondary battery as a power supply, it has a maximum load weight of about $6.6 \mathrm{~kg}$ and can fly for 11 minutes. The landing gear can be lifted while flight not to interfere with the light source attached to the bottom of the UAV. By folding the propeller arms of the UAV, the width, depth and height of it are about $400 \times 400 \times 500 \mathrm{~mm}$ respectively, and it can be put in a cargo room of a car.

[High accuracy GPS] The GPS installed in the UAV is used for control and can not record GPS coordinate information at flight. Also, the position determination accuracy using the GPS installed in UAV is $0.5 \mathrm{~m}$ in the vertical direction and $1.5 \mathrm{~m}$ in the horizontal direction. The required position accuracy is $\pm 0.1 \mathrm{~m}$ at $100 \mathrm{~m}$ ahead of FDs. Therefore, we use high precision GPS (Swift Navigation, Piksi) using Real Time Kinematic (RTK) positioning (Fig. 3 ). The high precision GPS uses two GPS modules, and the relative distance between the two modules is determined with $\pm 0.1 \mathrm{~m}$.

[Light source] UV-LEDs (Roithner Lasertechnik, H2A1-H375-E) with a peak wavelength of $375 \mathrm{~nm}$ are used as a light source. The light source consists of 12 UV-LEDs attached on each side of the dodecahedron which is made of ultraviolet curable resin and shaped by 3D printer as shown in Fig. 4, and it is covered with a spherical diffuser made of acrylic resin to improve the spatial uniformity of the light intensity.

The stability of the light intensity is $\sim 0.1 \%$ measured in the laboratory. The temperature sensor is also implemented on Opt-copter and we also are planning on flight shot-by-shot monitor of the light intensity. The azimuthal dependence of the light intensity is $\pm 9 \% / 2 \pi$ and $0.5 \sim 4 \%$ per 15 degrees which is enough, considering the flight stability of \pm 7 degrees. 


\section{Test observation at Utah} follows.

We tested Opt-copter at the TA site in September 2016. The Observation condition is as

- Flight distance : $200 \mathrm{~m}$ from the center of FD mirror.

- Wind speed $<5 \mathrm{~m} / \mathrm{s}$.

- Flight time : 60 seconds for each FD.

- Target PMT : The standard PMT installed at the center of PMT cluster. (Red colored PMT in Fig.5)

Calibration procedure is as follows. Firstly Opt-copter flies into the F.O.V. of camera 7 as a standard camera, secondly flies into the other cameras F.O.V. and finally returns into the F.O.V. of the standard camera as shown in Fig. 5.

We evaluated the positioning accuracy of Opt-copter Figure 6 shows histograms of the azimuth angle and the elevation angle of Opt-copter observed by FD camera 7 at the start and the end of the calibration procedure. The flight accuracy at the start of the calibration procedure was $-0.06 \mathrm{~m}$ in azimuth and $-0.02 \mathrm{~m}$ in elevation, and stability was $0.07 \mathrm{~m}$ in azimuth and $0.05 \mathrm{~m}$ in elevation. In the end of the procedure, the flight accuracy was -0.33 $\mathrm{m}$ in azimuth and $-0.40 \mathrm{~m}$ in elevation, and stability was $0.10 \mathrm{~m}$ in azimuth and $0.13 \mathrm{~m}$ in elevation.

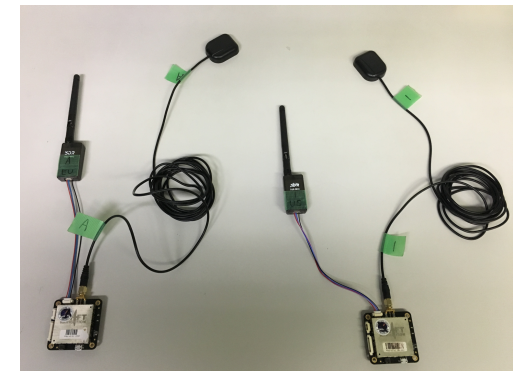

Fig. 3. High accuracy GPS. One is installed at the well known point and the other is mounted on Opt-copter.

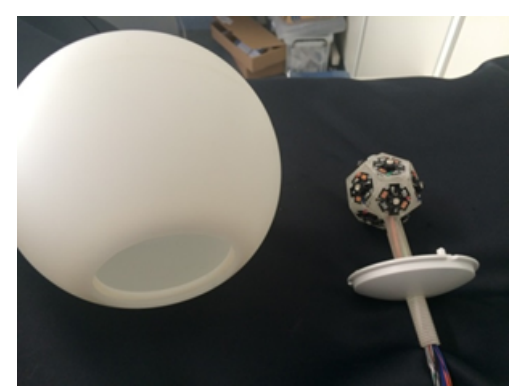

Fig. 4. UV-LEDs installed on the dodecahedron and Diffuser.

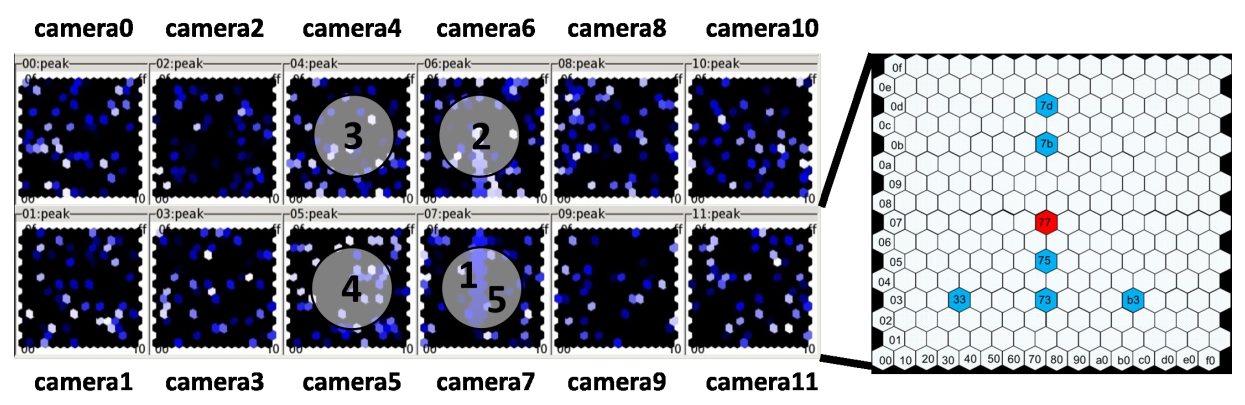

Fig. 5. FD F.O.V. of each camera and PMT address. The numbers of $1 \sim 5$ are example of the order of calibration procedure. 

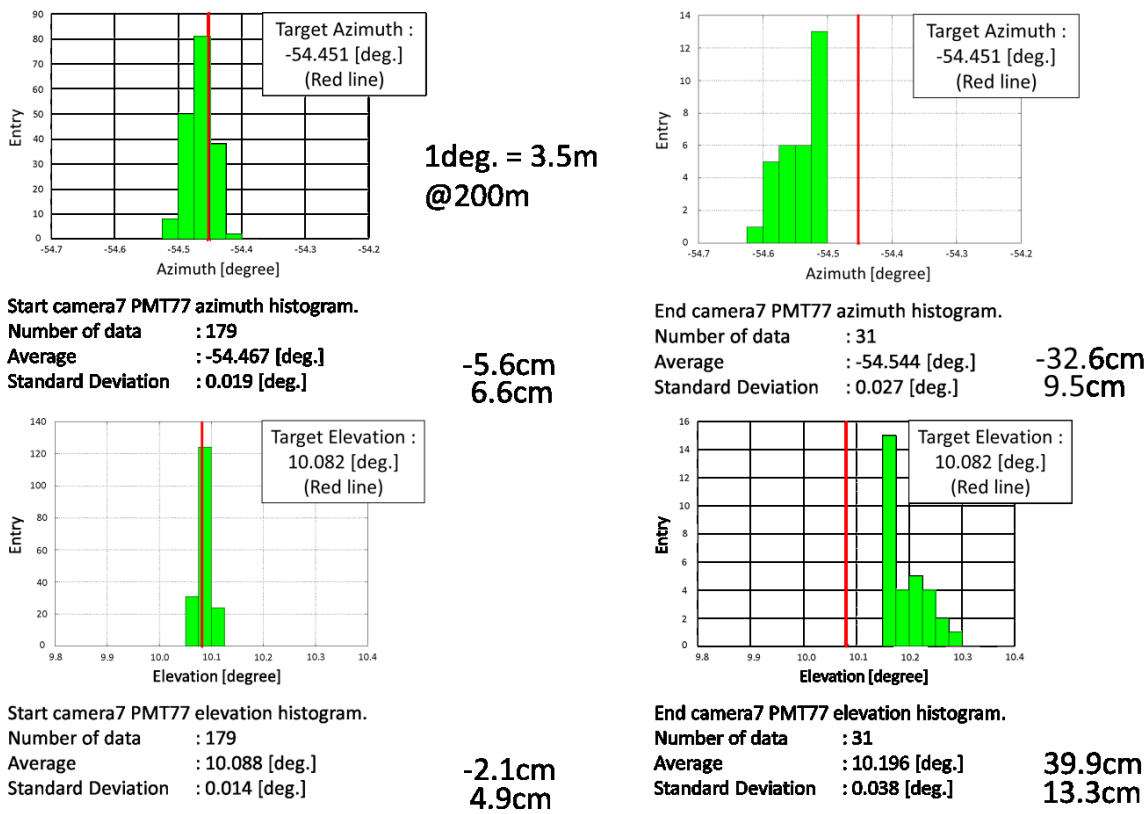

\begin{tabular}{lll}
\multicolumn{2}{l}{ Start camera7 PMT77 } & elevation histogram. \\
Number of data & $: 179$ & \\
Average & $: 10.088[\mathrm{deg}]$. & $-2.1 \mathrm{~cm}$ \\
Standard Deviation & $: 0.014[\mathrm{deg}]$. & $\mathbf{4 . 9} \mathrm{cm}$
\end{tabular}

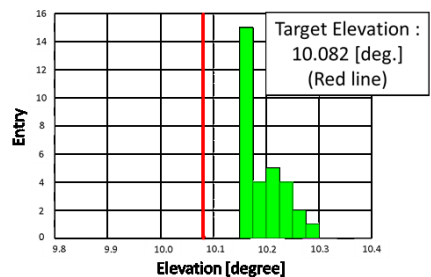

End camera7 PMT77 elevation histogram. Number of data $\quad: 31$ Average : 10.196 [deg.] $\quad 39.9 \mathrm{~cm}$ Standard Deviation : 0.038 [deg.] $\quad 13.3 \mathrm{~cm}$

Fig. 6. The histograms of azimuth and elevation angle of Opt-copter observed by FD, while Optcopter is in the F.O.V. of the standard PMT of camera 7. Left and right sides are the initial and last in a calibration procedure.

\section{Conclusion}

We tested Opt-copter at the TA site in September 2016 and succeeded in observing Opt-copter signal by the FDs self-trigger without saturating. At present we are comparing the data and simulation. Also, we're going to make the light source of more appropriate intensity. In the future, by using Opt-copter we will compare the detection efficiency of the FDs directly although the FDs are located separately. We can also expect to apply Opt-copter to calibrating various optical detectors installed outdoors.

\section{Acknowledgments}

The Telescope Array experiment is supported by the Japan Society for the Promotion of Science through Grants-in-Aids for Scientific Research on Specially Promoted Research (21000002) "Extreme Phenomena in the Universe Explored by Highest Energy Cosmic Rays" and for Scientific Research (19104006), and the Inter-University Research Program of the Institute for Cosmic Ray Research; by the U.S. National Science Foundation awards PHY0307098, PHY-0601915, PHY-0649681, PHY-0703893, PHY-0758342, PHY-0848320, PHY1069280, PHY-1069286, PHY-1404495 and PHY-1404502; by the National Research Foundation of Korea (2007-0093860, R32-10130, 2012R1A1A2008381, 2013004883); by the Russian Academy of Sciences, RFBR grants 11-02-01528a and 13-02-01311a (INR), IISN project No. 4.4509.10 and Belgian Science Policy under IUAP VII/37 (ULB). The foundations of Dr. Ezekiel R. and Edna Wattis Dumke, Willard L. Eccles and the George S. and Dolores Dore Eccles all helped with generous donations. The State of Utah supported the project through its Economic Development Board, and the University of Utah through the Office of the Vice President for Research. The experimental site became available through the cooperation of 
the Utah School and Institutional Trust Lands Administration (SITLA), U.S. Bureau of Land Management, and the U.S. Air Force. We also wish to thank the people and the officials of Millard County, Utah for their steadfast and warm support. We gratefully acknowledge the contributions from the technical staffs of our home institutions. An allocation of computer time from the Center for High Performance Computing at the University of Utah is gratefully acknowledged.

\section{References}

[1] H.Tokuno, et. al., NIM A, 676, 54-65 (2012), T.Abu-Zayyad et. al., NIM A, 689, 87-97 (2012), R.U. Abbasi et. al., Astropart. Physics, 68, 27-44 (2015).

[2] J.Parrisius, thesis, KIT (2009), F.Werner, thesis, KIT (2010).

[3] K.Machida, et. al., Proc. of 33rd ICRC, (2013). 